\title{
LP-MOVPE growth and properties of high Si-doped InGaAs contact layer for quantum cascade laser applications
}

\author{
B. ŚCIANA ${ }^{1 *}$, M. BADURA ${ }^{1}$, W. DAWIDOWSKI ${ }^{1}$, K. BIELAK $^{1}$, D. RADZIEWICZ1 ${ }^{1}$ D. PUCICKI ${ }^{1}$, \\ A. SZYSZKA ${ }^{1}$, K. ŻELAZNA ${ }^{2}$, and M. TŁACZAŁA ${ }^{1}$ \\ ${ }^{1}$ Faculty of Microsystem Electronics and Photonics, Wrocław University of Technology, \\ ul. Janiszewskiego 11/17, 50-372 Wroclaw, Poland \\ ${ }^{2}$ Laboratory for Optical Spectroscopy of Nanostructures, Department of Experimental Physics, \\ Wrocław University of Technology, ul. Wybrzeże Wyspiańskiego 27, 50-370 Wrocław, Poland
}

\begin{abstract}
The work presents doping characteristics and properties of high Si-doped InGaAs epilayers lattice-matched to InP grown by low pressure metal-organic vapour phase epitaxy. Silane and disilane were used as dopant sources. The main task of investigations was to obtain heavily doped InGaAs epilayers suitable for usage as plasmon-confinement layers in the construction of mid-infrared InAlAs/InGaAs/InP quantum-cascade lasers (QCLs). It requires the doping concentration of $1 \times 10^{19} \mathrm{~cm}^{-3}$ and $1 \times 10^{20} \mathrm{~cm}^{-3}$ for lasers working at $9 \mu \mathrm{m}$ and $5 \mu \mathrm{m}$, respectively. The electron concentration increases linearly with the ratio of gas-phase molar fraction of the dopant to III group sources (IV/III). The highest electron concentrations suitable for InGaAs plasmon-contact layers of QCL was achieved only for disilane. We also observed a slight influence of the ratio of gas-phase molar fraction of V to III group sources (V/III) on the doping efficiency. Structural measurements using high-resolution X-ray diffraction revealed a distinct influence of the doping concentration on InGaAs composition what caused a lattice mismatch in the range of $-240 \div-780$ ppm for the samples doped by silane and disilane. It has to be taken into account during the growth of InGaAs contact layers to avoid internal stresses in QCL epitaxial structures.
\end{abstract}

Keywords: InGaAs/InP heterostructures, silicon-doping, InGaAs plasmon-contact layer, quantum cascade lasers.

\section{Introduction}

The unipolar quantum cascade lasers are the novel coherent light sources working in the mid-IR, far-IR and terahertz spectral ranges. Their emission based on inter-subband electron transitions inside quantum wells depends on the thickness of the constituent layers and is not determined by the bandgap of the used materials as in the case of typical semiconductor bipolar lasers. QCLs have the large number of applications in high-resolution molecular spectroscopy, industrial control, medical diagnostics, military and law enforcement and free-space telecommunications. Since the first demonstration in 1994 [1], they have undergone many improvements to achieve higher continuous-wave powers, better temperature stability and lower threshold currents $[2,3]$.

This work presents the doping characteristics and properties of high Si-doped InGaAs epilayers $\left(\mathrm{n}^{++}\right.$-InGaAs) lattice-matched to InP grown by low pressure metal organic vapour phase epitaxy (LP-MOVPE) using silane and disilane as dop-

\footnotetext{
*e-mail: beata.sciana@pwr.edu.pl
}

ant sources. The studies were focused on obtaining the high Si-doped InGaAs working as a plasmon-confinement layer in a short-wavelength mid-infrared $(\sim 4.7 \mu \mathrm{m})$ QCL based on the strain-compensated InGaAs/InAlAs active region [4,5]. Due to a narrow bandgap, small thermal resistivity and easy to get ohmic contact InGaAs is usually applied as a contact layer of QCLs based on InGaAs/InAlAs/InP heterostructures. Additionally, the anomalous dielectric dispersion near the plasma frequency is achieved in this semiconductor, even for lower doping levels $\left(<10^{20} \mathrm{~cm}^{-3}\right)$ than in the case of InP what allows to improve the laser performance working at shorter wavelengths $(<10 \mu \mathrm{m})$. The crucial role of a heavily doped $\mathrm{n}^{++}$-InGaAs layer is to suppress the coupling between the fundamental waveguide mode and the high-loss plasmon mode propagating along the metal contact-semiconductor interface. The doping concentration in the range of $10^{19} \div 10^{20} \mathrm{~cm}^{-3}$ is enough to decrease the InGaAs refractive index near the plasma frequency what significantly enhances the confinement factor of the waveguide mode $[4,6]$.

In this work we study the influence of the growth parameters as growth rate (GR), IV/III and V/III ratios on the electron 
concentration in Si-doped InGaAs epilayers grown by LP-MOVPE using silane and disilane as dopant sources. The properties of the investigated structures were measured using high-resolution X-ray diffraction (HRXRD), photoluminescence (PL) spectroscopy, electrochemical capacitance-voltage $(\mathrm{EC}-\mathrm{V})$ profiling and atomic force microscopy (AFM).

\section{Experimental details}

Test structures, consisted of a $200 \mathrm{~nm}$ thick Si-doped InP buffer and $250 \div 600 \mathrm{~nm}$ thick Si-doped InGaAs layers, were grown on (100)-oriented sulphur-doped InP substrates by LP-MOVPE. Additionally, the undoped reference structures consisted of an InP buffer, InGaAs layer and InP "cap" with thicknesses of 200, 250 and $30 \mathrm{~nm}$, respectively were deposited (samples P69, P88). Trimethylgallium (TMGa), trimethylindium (TMIn), phosphine $\left(100 \% \mathrm{PH}_{3}\right)$, arsine $\left(100 \% \mathrm{AsH}_{3}\right)$, silane (200 ppm $\mathrm{SiH}_{4}$ in $\mathrm{H}_{2}$ ), and disilane (100 ppm $\mathrm{Si}_{2} \mathrm{H}_{6}$ in $\mathrm{H}_{2}$ ) were used as growth and dopant precursors. The studies focused on obtaining the high-doped InGaAs epilayers ( $\mathrm{n}=$ $10^{19} \div 10^{20} \mathrm{~cm}^{-3}$ ) suitable for usage as plasmon-confinement layers in the construction of QCL working at $4.7 \mu \mathrm{m}$. The waveguide core of the mentioned laser, based on the strain-compensated $\mathrm{In}_{0.669} \mathrm{Ga}_{0.331} \mathrm{As} / \mathrm{In}_{0.362} \mathrm{Al}_{0.638} \mathrm{As}$ heterostructure, will be made by $\mathrm{MBE}$ while the $\mathrm{InP}$ waveguide claddings and $\mathrm{n}^{++}-$InGaAs contact layer will be grown using LP-MOVPE. This hybrid technology excludes applying of the high growth temperatures for increasing the electron concentrations in the $\mathrm{n}^{++}$-InGaAs top layer, what simultaneously avoids deterioration of the core region made by MBE technique. Thus, during the growth processes the following parameters, elaborated in our earlier studies for undoped UD-InGaAs epilayers, were constant [7]: growth temperature $\mathrm{T}_{\mathrm{g}}=635^{\circ} \mathrm{C}$, reactor pressure $\mathrm{p}=100 \mathrm{mbar}$, and gas-phase molar ratio $\mathrm{Ga} / \mathrm{III}=0.36$. They guarantee high material quality of UD-InGaAs lattice-matched to InP. The doping concentration in $\mathrm{n}^{++}$-InGaAs was controlled by changing the growth rate $\mathrm{GR}=1.08 \div 3.12 \mu \mathrm{m} / \mathrm{h}$ and the gas-phase molar ratios $\mathrm{IV} / \mathrm{III}=0.16 \div 3.28 \%$ and $\mathrm{V} / \mathrm{III}=26 \div 240$. The doping characteristics and properties of the investigated structures are presented for silane (samples P54 $\div$ P57) and disilane (samples

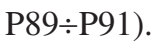

\section{Results and discussion}

\subsection{Doping characteristics}

The free electron distribution across the investigated structures was measured using a Bio-Rad PN 4300 electrochemical capacitance-voltage (EC-V) profiler at a frequency range of $0.3 \div 3 \mathrm{kHz}$. This method applies the capacitance-voltage analysis of a reverse bias electrolyte-semiconductor Schottky junction for evaluation of a free carrier concentration. Firstly, the

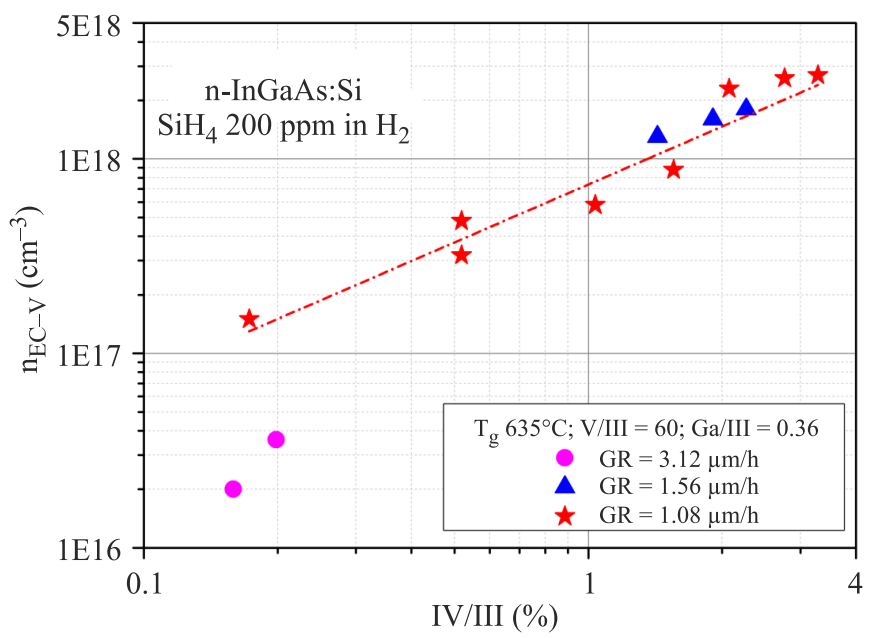

(a)

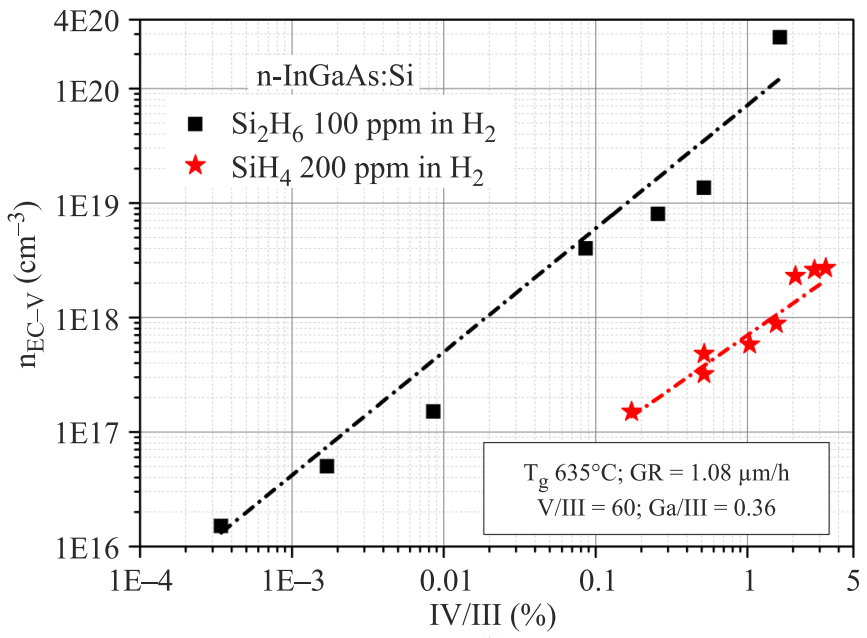

(b)

Fig. 1. The dependence of the electron concentration on the gas-phase molar ratio IV/III for different $\mathrm{Si}$ dopant sources: (a) silane $\mathrm{SiH}_{4}$ (200 ppm in $\mathrm{H}_{2}$ ); (b) disilane $\mathrm{Si}_{2} \mathrm{H}_{6}\left(100 \mathrm{ppm}\right.$ in $\mathrm{H}_{2}$ ).

doping efficiency of InGaAs epilayers was investigated using silane as a dopant source. The following parameters were kept constant: process temperature $635^{\circ} \mathrm{C}, \mathrm{Ga} / \mathrm{III}=0.36$ what guaranteed the growth of InGaAs lattice-matched to $\operatorname{InP}(\sim 53 \%$ of InAs mole fraction), $\mathrm{V} / \mathrm{III}=60$. The dependence of electron concentration on the ratio of gas-phase molar fraction of silane to III group sources (IV/III ratio) at different growth rates is shown in Fig. 1(a). The growth rate was controlled by changing the concentration of III group sources in the reactor atmosphere. The highest electron concentration of $2.7 \times 10^{18} \mathrm{~cm}^{-3}$ was achieved for IV/III $=3.28 \%$ after lowering the growth rate from 3.12 to $1.08 \mu \mathrm{m} / \mathrm{h}$ (III group sources flow rate was decreased from 74.92 to $25.84 \mu \mathrm{mol} / \mathrm{min}$ ). It suggests that $\mathrm{Si}$ incorporation into InGaAs takes place via vacant sites of III group elements as in the case of Si-doped GaAs and InP $[8,9]$. 
The electron concentration increases linearly with the IV/III ratio (the slope is 0.93 ) independent on the growth rates for 1.08 and $1.56 \mu \mathrm{m} / \mathrm{h}$. In further studies disilane was used as a dopant source to exceed the doping level of $10^{19} \mathrm{~cm}^{-3}$ suitable for future applications in QCLs. Compared to silane, disilane decomposes at lower temperatures due to the weaker $\mathrm{Si}-\mathrm{Si}$ bond $(226 \mathrm{~kJ} / \mathrm{mol})$ in $\mathrm{Si}_{2} \mathrm{H}_{6}$ than the $\mathrm{Si}-\mathrm{H}$ bond in $\mathrm{SiH}_{4}$ $(318 \mathrm{~kJ} / \mathrm{mol})$ [10] what gives a higher doping efficiency. The influence of IV/III ratio on the electron concentration in InGaAs epilayers for disilane and the lowest growth rate $(1.08 \mu \mathrm{m} / \mathrm{h})$ is presented in Fig. 1(b). To compare two kinds of dopant sources the same dependence for silane was included. The relationship for disilane is linear in the range of carrier concentration of $10^{16} \div 10^{20} \mathrm{~cm}^{-3}$ with the slope slightly greater (1.08) than in the case of silane. The doping efficiency about two orders of magnitude higher than for silane allows to obtain heavily doped $\mathrm{n}^{++}$-InGaAs $\left(\mathrm{n}_{\max } \sim 2.8 \times 10^{20} \mathrm{~cm}^{-3}\right.$ for $\mathrm{IV} / \mathrm{III}=1.64 \%$ ) that can be used as a plasmon-confinement layer in QCLs working at $4.7 \mu \mathrm{m}$. The achieved maximum value of the electron concentration in Si-doped InGaAs epilayers has not been previously published.

The silicon incorporation into crystalline structure of III-V semiconductor compounds may also depend on V/III ratio, especially for the high doping levels. The influence of V/III ratio on Si-doped InGaAs using disilane was investigated for the growth conditions corresponding to the highest electron concentration $(\mathrm{GR}=1.08 \mu \mathrm{m} / \mathrm{h}, \mathrm{IV} / \mathrm{III}=1.64 \%)$. The ratio of V/III was controlled by changing the flow rate of $\mathrm{AsH}_{3}$. The dependence of Si doping in InGaAs on the V/III ratio varied in the range of $26 \div 240$ what is shown in Fig. 2. It is visible that the highest electron concentration was achieved for $\mathrm{V} / \mathrm{III}=60$ founded in our earlier studies. For lower values of V/III ratio (lower $\mathrm{AsH}_{3}$ flow rates) the electron concentration decreases what can be explained by amphoteric behaviour of $\mathrm{Si}$ and a greater tendency to occupy As sites. The reduction

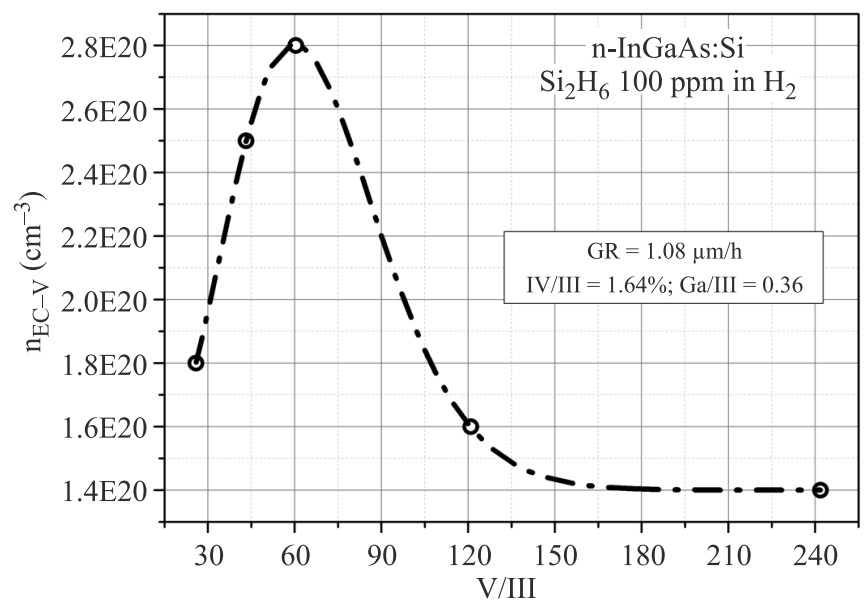

Fig. 2. The dependence of the electron concentration in Si-doped InGaAs on the V/III ratio. of doping efficiency for higher $\mathrm{AsH}_{3}$ concentration in a gas phase $(\mathrm{V} / \mathrm{III}>60)$ results probably from the high surface coverage of As species what decreases the amount of vacant silylene $\mathrm{SiH}_{2}$ adsorption sites and thus $\mathrm{Si}$ incorporation into InGaAs. This corresponds to the model proposed by Field and Ghandi [9].

\subsection{Influence of doping concentration on the lattice mismatch of Si-doped InGaAs to InP - HRXRD analysis}

Structural properties and the lattice mismatch $(\Delta \mathrm{a} / \mathrm{a}=$ $\left.\left(\mathrm{a}_{\text {InP }}-\mathrm{a}_{\text {InGaAs }}\right) / \mathrm{a}_{\text {InP }}\right)$ of Si-doped InGaAs epilayers to InP substrate were analyzed by a high-resolution X-ray diffraction (HRXRD). Measured diffraction curves of the (004) reflection in the triple axis geometry obtained for Si-doped InGaAs using silane and disilane are shown in Fig. 3(a) (samples

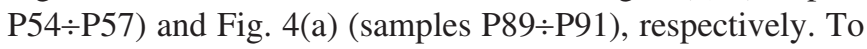
check the influence of Si-doping on an InGaAs lattice parameter, the rocking curves measured for the undoped samples were also included (samples P69 and P88). The peak with the

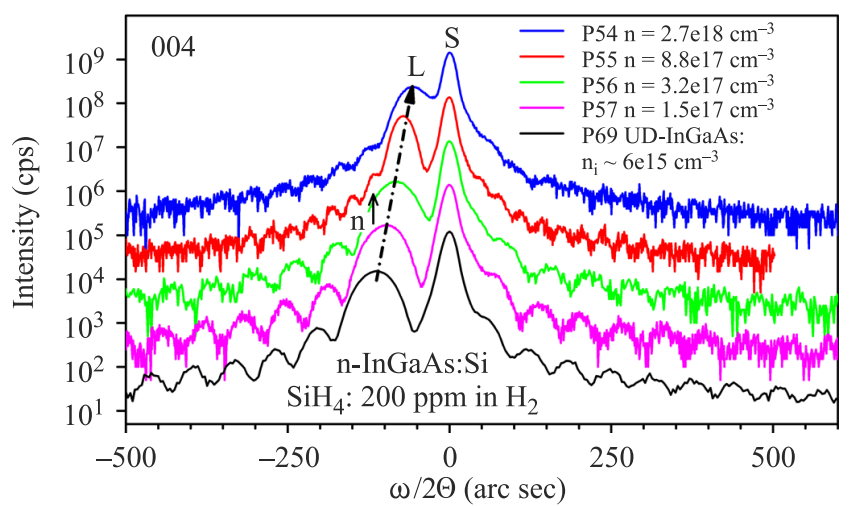

(a)

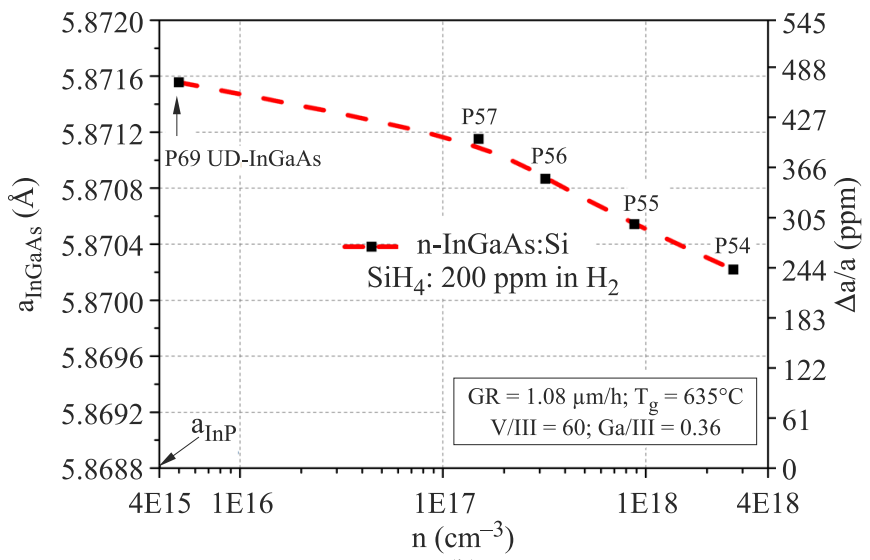

(b)

Fig. 3. Influence of the electron concentration in Si-doped InGaAs by silane on: (a) the measured diffraction curves; (b) the lattice parameter and lattice mismatch to InP. 


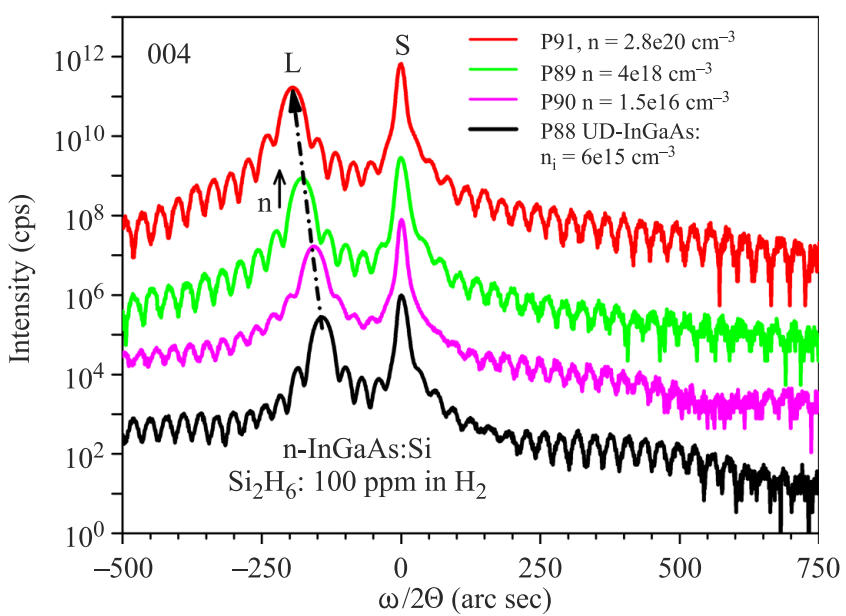

(a)

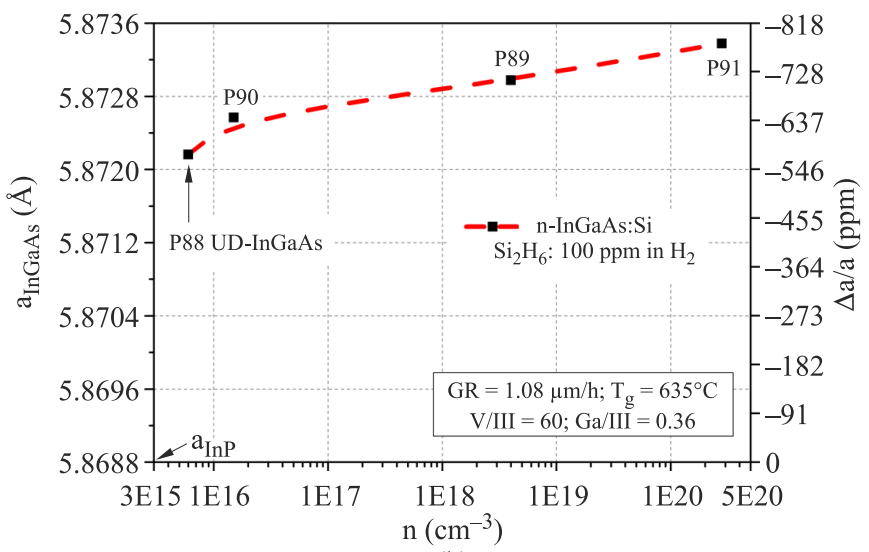

(b)

Fig. 4. Influence of the electron concentration in Si-doped InGaAs by disilane on: (a) the measured diffraction curves; (b) the lattice parameter and lattice mismatch to InP.

highest intensity comes from InP substrate (S) while the broad and weaker peak visible on the left corresponds to InGaAs epilayer (L). The presence of the Pendellösung fringes for all measured samples indicates their good crystalline quality and the sharpness of the InGaAs/InP interface. In the silane case the lattice parameter of the Si-doped InGaAs epilayer decreases with the carrier concentration changed from $1.5 \times 10^{17}$ to $2.7 \times 10^{18} \mathrm{~cm}^{-3}$ (the layer peak L shifts towards the substrate peak S) what results in decreasing the lattice mismatch of InGaAs to InP of about 228 ppm [Fig. 3(b)]. The mentioned effect can be explained by a smaller ionic radius of the Si dopant than substituted III group host atoms (Ga and In) or a greater tendency of Si to occupy In sites.

The situation is different in the case of a disilane dopant source. The lattice parameter of Si-doped InGaAs epilayers deposited at the same growth conditions increases with the electron concentration changed from $1.5 \times 10^{16}$ to $2.8 \times 10^{20} \mathrm{~cm}^{-3}$. The layer peak L moves away from the substrate peak $\mathrm{S}$ to the left (i.e., to the lower angles), what is visi- ble in Fig. 4(a). It can be connected with a greater tendency of Si to occupy Ga sites (in contrast to silane) what increases In content in InGaAs and thus the lattice mismatch to $\mathrm{InP}$ of about 207 ppm [Fig. 4(b)].

This behaviour of Si incorporated into an InGaAs crystalline structure is well explained by theoretical model presented by Cheng-Wei Lee et al. [11]. They found that the smallest formation energy $(0.15 \div 0.95 \mathrm{eV}$ depending on the atomic ordering) has the substitutional defect created by Si replacing an In atom in the InGaAs lattice, but it can introduce significant strain energy into the system. In the case of $\mathrm{Si}$ atoms introduced into $\mathrm{Ga}$ sites the formation energy is slightly higher $(0.57 \div 1.22 \mathrm{eV})$. When In and $\mathrm{Ga}$ atoms are randomly arranged in InGaAs matrix, there is no preference for $\mathrm{Si}$ to replace either an In or a $\mathrm{Ga}$ atom on a lattice site. It means that minor differences between the amount of Si atoms that occupy In and $\mathrm{Ga}$ sites can increase or decrease a lattice parameter of Si-doped InGaAs. Of course, there is also possible an interstitial location of Si atoms in InGaAs what can increase its lattice parameter but the probability of this situation is low due to the substantially higher formation energy of these interstitial defects $(1.22 \div 7.77 \mathrm{eV}$ depending on atomic ordering $)$ in comparison to the substitutional Si incorporation [11].

\subsection{Influence of doping concentration on PL spectra of Si-doped InGaAs}

The heavy doping of semiconductor materials distinctly influences on their band structure. One of changes is the effective bandgap narrowing (BGN) due to the both the band tailing and many-body effects (mainly electron-electron and electron-impurity interactions) [12]. The inhomogeneous impurity distribution destroys also the translational symmetry of the crystal so the indirect transitions are also possible. Additionally, the heavily n-doped semiconductors reveal an increase in the interband transition energy due to the filling of the conduction band with electrons (the rise of the Fermi level above the conduction-band edge), what is known as the Burstein-Moss (BM) effect $[13,14]$. The mentioned changes are responsible for the optical bandgap shift $\Delta E_{g}$. For a single parabolic conduction band this parameter is given by Ref. 15

$$
\Delta E_{g}=E_{g_{\text {opt }}}-E_{g_{0}}=E_{F}-E_{b g n}=\left(\frac{\hbar^{2}}{2 m^{*}}\right)\left(3 \pi^{2} N\right)^{2 / 3}-E_{b g n},
$$

where $E_{\text {gopt }}$ is the effective optical bandgap (the position of the Fermi level above the valence band), $E_{g 0}$ is the nominal semiconductor bandgap (undoped semiconductors), $E_{F}$ is the position of the Fermi level above the conduction band (BM effect), $\hbar$ is the reduced Planck's constant, $m^{*}$ is the conduction band effective mass, $N$ is the electrically active carrier 
concentration, and $E_{b g n}$ is the effective bandgap narrowing. In the case of a conduction band nonparabolicity, the first term in the final version of Eq. (1) is usually modified to take into account the conduction effective mass dependence on the carrier concentration. Influence of Si-doping on the InGaAs epilayers on their optical properties was examined using photoluminescence spectroscopy. The measurements were carried out at $295 \mathrm{~K}$ and $20 \mathrm{~K}$ using InGaAs single-channel detector and YAG laser operating at $532 \mathrm{~nm}$ with the optical power of $100 \mathrm{~mW}$. The exemplary PL spectra obtained for the undoped (sample P88) and Si-doped (sample P89, $\mathrm{n}=4 \times 10^{18} \mathrm{~cm}^{-3}$ ) InGaAs epilayers are presented in Fig. 5. The bandgap energy was determined as the photoluminescence peak energy minus $\mathrm{kT} / 2$. With an increase in doping concentration, the intensity of emission lines decreases, PL peaks become broad and shift towards higher energy. A blue shift of the peaks' position is connected with the Burstein-Moss effect. The influence of the electron concentration on the obtained PL spectra is well visible at low temperature (blue lines in Fig. 5). The strong and

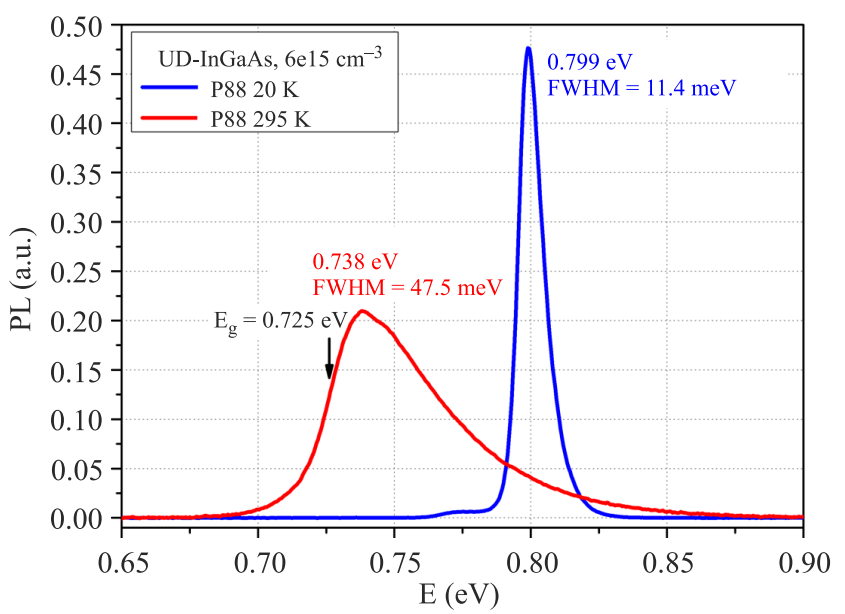

(a)

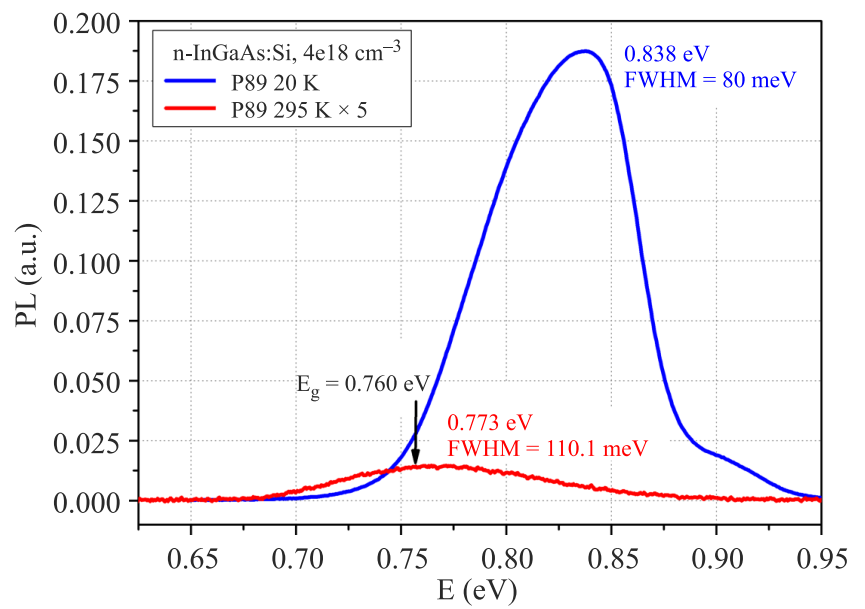

(b)

Fig. 5. PL spectra measured at $295 \mathrm{~K}$ and $20 \mathrm{~K}$ of: (a) the undoped InGaAs (sample P88); (b) the high Si-doped InGaAs (sample P89). symmetric excitonic line at $0.799 \mathrm{eV}$ obtained for the undoped sample P88 becomes a wide and asymmetric emission spectrum for a Si-doped sample P89 with a steeper slope on the high-energy side in comparison with the slope on the low-energy side.

The dependence of the bandgap shift $\left(\Delta E_{g}\right)$ of the investigated $\mathrm{Si}$-doped InGaAs epilayers on electron concentration changed in the range of $1.5 \times 10^{16} \div 4 \times 10^{18} \mathrm{~cm}^{-3}$ is shown in Fig. 6. The solid red and open blue circles correspond to the PL peak position shifts at $295 \mathrm{~K}$ and $20 \mathrm{~K}$, respectively. For $\mathrm{n}>4 \times 10^{18} \mathrm{~cm}^{-3}$ the PL spectra were significantly distorted and precise determination of the bandgap shift was difficult.

The experimental results are compared with theoretical calculations of the Burstein-Moss shift $\Delta E_{B M}$ (dashed line in Fig. 6) and with the bandgap shift caused by both bandgap widening due to the conduction band filling and bandgap narrowing $\Delta E_{g}=\Delta E_{B M}-\Delta E_{b g n}$ (dash-dotted line in Fig. 6). The value of $\Delta E_{B M}$ was calculated in pursuance of parabolic-band theory using Eq. (1) $-E_{F}$ parameter, taking into account the effect of a nonparabolic conduction band by introducing the doping dependent electron effective mass $m_{e}{ }^{*}$ according to the third order polynomial empirical model (Poly3x) proposed by James C. Li et al. [16]

$$
\frac{m_{e}^{*}}{m_{0}}=A N_{D}^{3}+B N_{D}^{2}+C N_{D}+D,
$$

where $m_{e}^{*}$ is the electro effective mass, $m_{0}$ is the free electron rest mass, $N_{D}$ is the total donor concentration, $A, B, C, D$ are

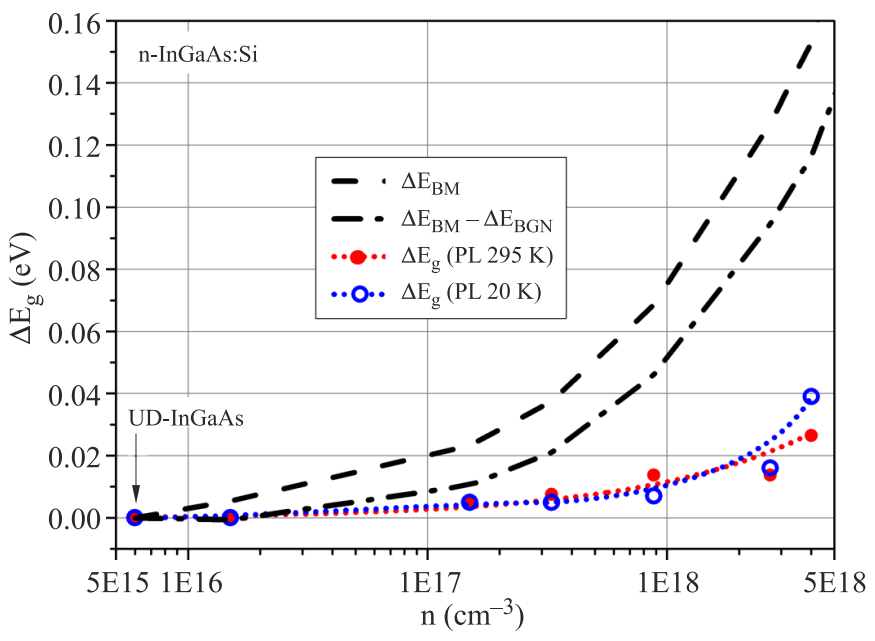

Fig. 6. The dependence of the bandgap shift $\Delta E_{g}$ of the investigated Si-doped InGaAs epilayers on the electron concentration determined from PL spectra performed at $295 \mathrm{~K}$ and $20 \mathrm{~K}$ (solid red and open blue circles, respectively) and from theoretical calculation of the Burstein-Moss shift $\Delta E_{B M}$ (dashed line) and the bandgap shift caused by the both the bandgap widening due to the conduction band filling and the bandgap narrowing $\Delta E_{B M}-\Delta E_{b g n}$ (dash-dotted line). 
the polynomial coefficients $\left(7.8474 \times 10^{-3},-4.1373 \times 10^{-1}\right.$, 7.2756 and -42.629 , respectively, for InGaAs lattice-matched to InP). The value of the bandgap narrowing $\Delta E_{b g n}$ was calculated considering the electron-electron interaction [17]

$$
\Delta E_{b g n}=2.03 \times 10^{-8} n^{1 / 3},
$$

where $n$ is the electron concentration in $\mathrm{n}$-doped InGaAs.

From Fig. 6 it is seen that the bandgap shift $\Delta E_{g}$ of Si-doped InGaAs determined from PL spectra is negligible for the electron concentrations below $3.3 \times 10^{17} \mathrm{~cm}^{-3}$ and slightly increases at $\mathrm{n}=10^{18} \mathrm{~cm}^{-3}$ reaching the values of $26.5 \mathrm{meV}$ (at $295 \mathrm{~K}$ ) and $39 \mathrm{meV}$ (at $20 \mathrm{~K}$ ) for electron concentration $4 \times 10^{18} \mathrm{~cm}^{-3}$. Obtained values of $\Delta E_{g}$ are lower than theoretical calculations and discrepancy rises with the doping concentration to $80 \mathrm{meV}$ for $\mathrm{n}=4 \times 10^{18} \mathrm{~cm}^{-3}$ and about $237 \mathrm{meV}$ for the highest electron concentration achieved in this work $\left(2.8 \times 10^{20} \mathrm{~cm}^{-3}\right)$. Such differences between experimental results and theoretical predictions of the bandgap shift of heavily doped semiconductors were also reported by other authors $[17,18]$ what confirms the need of experimental works in this field to improve the existing theoretical models.

\subsection{Surface morphology of Si-doped InGaAs - AFM analysis}

The surface morphology of Si-doped InGaAs was examined using atomic force microscopy. The main aim of AFM measurements was determination of surface roughness of the heavily Si-doped InGaAs $\left(\mathrm{n}>10^{19} \mathrm{~cm}^{-3}\right)$ suitable for the usage as plasmon-contact layers in QCL constructions. Fig. 7 shows AFM images and the evaluated root mean square roughness

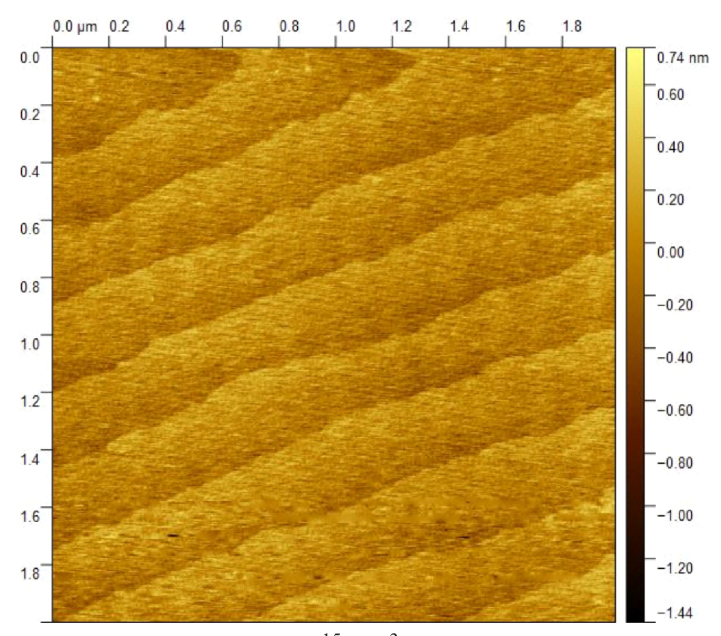

UD-InGaAs, $\mathrm{n}_{\mathrm{i}}=6 \times 10^{15} \mathrm{~cm}^{-3}$, RMS $=0.147 \mathrm{~nm}$

(a)

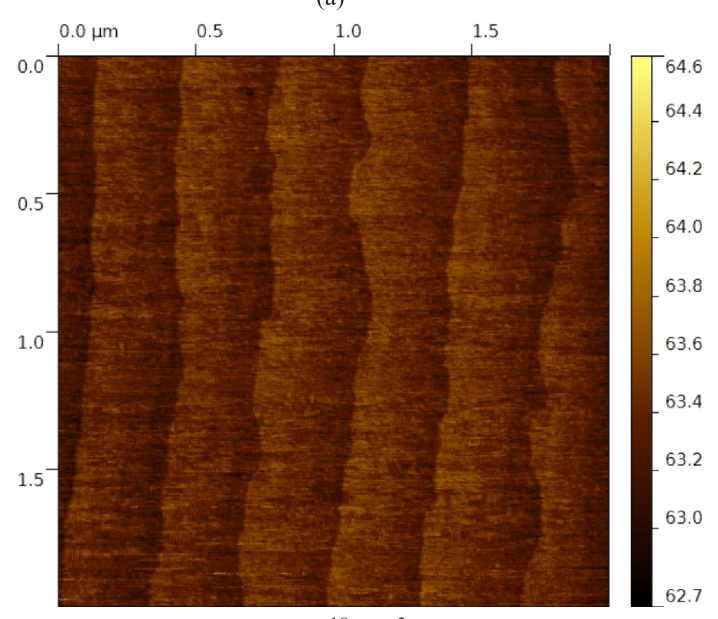

$\mathrm{n}^{+}-\mathrm{InGaAs}: \mathrm{Si}, \mathrm{n}=2.7 \times 10^{18} \mathrm{~cm}^{-3}, \mathrm{RMS}=0.133 \mathrm{~nm}$

(c)

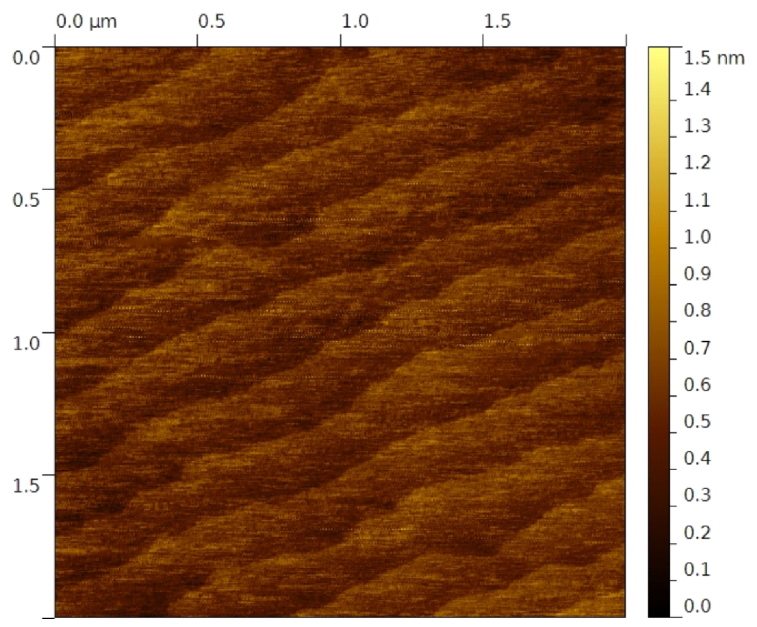

n-InGaAs:Si, $\mathrm{n}=3.2 \times 10^{17} \mathrm{~cm}^{-3}, \mathrm{RMS}=0.125 \mathrm{~nm}$

P56

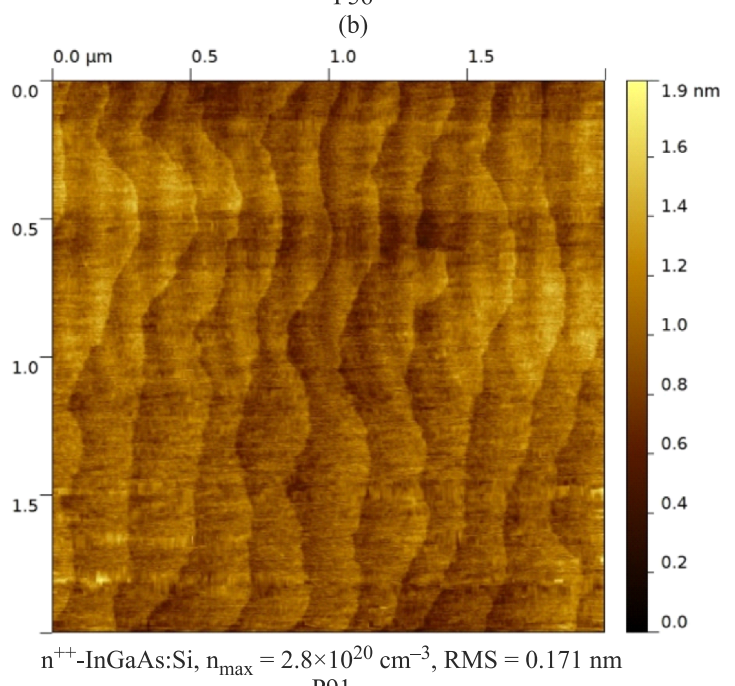

P9

(d)

Fig. 7. AFM images and RMS parameters of: (a) the undoped InGaAs - sample P69; (b), (c) and (d) Si-doped InGaAs with different electron concentrations: $3.2 \times 10^{17} \mathrm{~cm}^{-3}$ - sample P56, $2.7 \times 10^{18} \mathrm{~cm}^{-3}$ - sample P54, and $2.8 \times 10^{20} \mathrm{~cm}^{-3}$ - sample P91, respectively. (AFM scan area: $2 \mu \mathrm{m} \times 2 \mu \mathrm{m})$. 
Table 1. RMS parameters of the Si-doped InGaAs with different electron concentrations. The undoped sample is included as a reference structure.

\begin{tabular}{lcc}
\hline Structure (sample) & $\begin{array}{c}\text { Electron concentration n } \\
\left(\mathrm{cm}^{-3}\right)\end{array}$ & RMS (nm) \\
\hline UD-InGaAs (P69) & $6.0 \times 10^{15}$ & 0.147 \\
n-InGaAs:Si (P90) & $1.5 \times 10^{16}$ & 0.158 \\
n-InGaAs:Si (P57) & $1.5 \times 10^{17}$ & 0.118 \\
n-InGaAs:Si (P56) & $3.2 \times 10^{17}$ & 0.125 \\
n-InGaAs:Si (P55) & $8.8 \times 10^{17}$ & 0.137 \\
n-InGaAs:Si (P54) & $2.7 \times 10^{18}$ & 0.133 \\
n-InGaAs:Si (P89) & $4.0 \times 10^{18}$ & 0.130 \\
n-InGaAs:Si (P91) & $2.8 \times 10^{20}$ & 0.171 \\
\hline
\end{tabular}

(RMS) values for the undoped InGaAs with the intrinsic electron concentration $\mathrm{n}_{\mathrm{i}}=6 \times 10^{15} \mathrm{~cm}^{-3}$ (sample P69) and the $\mathrm{Si}$-doped InGaAs with the electron concentration varied from $3.2 \times 10^{17} \mathrm{~cm}^{-3}$ to $2.8 \times 10^{20} \mathrm{~cm}^{-3}$ (samples P56, P54, P91). The obtained results indicate that $\mathrm{Si}$-doping does not deteriorate a crystalline quality of InGaAs epilayers. The AFM image of the highest Si-doped sample P91 shows a step-flow growth mode and a slightly worse surface roughness (RMS = $0.171 \mathrm{~nm}$ ) in comparison to the undoped sample P69 (RMS = $0.147 \mathrm{~nm})$.

The RMS parameters evaluated for all investigated structures with the electron concentration changed in the range of $1.5 \times 10^{16} \div 2.8 \times 10^{20} \mathrm{~cm}^{-3}$ are listed in Table 1 (AFM scan area: $2 \mu \mathrm{m} \times 2 \mu \mathrm{m})$.

\section{Conclusions}

The frame growth parameters of the LP-MOVPE process allowing to obtain the heavily Si-doped $\mathrm{n}^{++}$-InGaAs layers suitable for application as plasmon-contact layers in QCLs were determined. The maximum value of the electron concentration of $2.8 \times 10^{20} \mathrm{~cm}^{-3}$ was achieved for the gas-phase molar ratios $\mathrm{IV} / \mathrm{III}=1.64 \%$ using disilane as a dopant source. Based on HRXRD measurements it was found that Si doping increases or decreases a lattice parameter of Si-doped InGaAs epilayers (depending on substitutional incorporation of $\mathrm{Si}$ atoms) what has to be taken into account during the QCLs fabrication to avoid the lattice strains introduced into the laser structure. The PL spectra showed an influence of the Burstein-Moss effect on the Si-doped InGaAs bandgap, especially for the highest doping levels. The bandgap shift is lower than the theoretical predictions and this discrepancy rises with the electron concentration up to $237 \mathrm{meV}$ for the highest doping level of $2.8 \times 10^{20} \mathrm{~cm}^{-3}$. The surface morphology of all investigated structures showed a step-flow growth mode and the surface roughness comparable with the undoped epilayers. The ob- tained results are fruitful in the point of view of the fundamental researches connected with the properties of heavily doped semiconductors and the application of Si-doped InGaAs in QCL constructions.

\section{Acknowledgements}

This work was supported by Wrocław University of Technology statutory grants, the Polish National Center for Research and Development under the projects: No. PBS1/B3/2/2012 "EDEN", No. PBS2/A3/15/2013 "PROFIT".

\section{References}

1. J. Faist, F. Capasso, D.L. Sivco, C. Sirtori, A.L. Hutchinson, and A.Y. Cho, "Quantum cascade laser", Science 264, 553-556 (1994).

2. C.A. Wang, R.K. Huang, A. Goyal, J.P. Donnelly, D.R. Calawa, S.G. Cann, F. O’Donnell, J.J. Plant, L.J. Missaggia, and G.W. Turner, "OMVPE growth of highly strain-balanced GaInAs/AlInAs/InP for quantum cascade lasers", J. Crystal Growth 310, 5191-5197 (2008).

3. D.G. Revin, J.P. Commin, S.Y. Zhang, A.B. Krysa, K. Kennedy, and J.W. Cockburn, "InP-based midinfrared quantum cascade lasers for wavelengths below $4 \mu \mathrm{m}$ ", IEEE J. Selected Topics in Quantum Electronics 17, 1417-1425 (2011).

4. C. Sirtori, J. Faist, F. Capasso, D.L. Sivco, A.L. Hutchinson, and A.Y. Cho, "Quantum cascade laser with plasmon-enhanced waveguide operating $8.4 \mu \mathrm{m}$ wavelength", Appl. Phys. Lett. 66, 3242-3244 (1995).

5. M. Bugajski, P. Gutowski, P. Karbownik, A. Kolek, G. Hałdaś, K. Pierściński, D. Pierścińska, J. KubackaTraczyk, I. Sankowska, A. Trajnerowicz, K. Kosiel, A. Szer- ling, J. Grzonka, K. Kurzydłowski, T. Slight, and W. Mere- dith, "Mid-IR quantum cascade lasers: Device technology and non-equilibrium Green's function modeling of electro-optical characteristics", Phys. Status Solidi B 251, 1144-1157 (2014).

6. T. Gresch, Gain and waveguide engineering in mid-infrared quantum cascade lasers, Doctoral Dissertation - Diss. ETH No. 18732, pp. 113-119, Zurich, 2009.

7. M. Badura, K. Bielak, B. Ściana, D. Radziewicz, D. Pucicki, W. Dawidowski, and M. Tłaczała, ,LP-MOVPE technology of InGaAs/GaAs heterostructures for application in Quantum Cascade Lasers", Elektronika 11, 34-36 (2014).

8. A.R. Clawson, T.T. Vu, and D.I. Elder, "A comparison of IV and VI n-dopants for MOVPE-grown InP", J. Crystal Growth 83, 211-218 (1987).

9. R.J. Field and S.K. Ghandhi, "Doping of gallium arsenide in a low pressure organometallic CVD system: I. Silane", J. Crystal Growth 74, 543-550 (1986).

10. F. Martin, Jean-Michel Hartmann, V. Carron, and Y. Le Tiec, Chemistry in Microelectronics, ISTE Ltd and John Wiley \& Sons, Inc., London, Hoboken, 2013.

11. L. Cheng-Wei, B. Lukose, M.O. Thompson, and P. Clancy, "Energetics of neutral Si dopants in InGaAs: An ab initio and 
semiempirical Tersoff model study", Physical Review B 91, 094108-1-094108-11 (2015).

12. H.C. Casey Jr. and F. Stern, "Concentration-dependent absorption and spontaneous emission of heavily doped GaAs", $J$. Appl. Phys. 47, 631-643 (1976).

13. E. Burstein, "Anomalous optical absorption limit in InSb", Phys. Rev. 93, 632-633 (1954).

14. T.S. Moss, "The interpretation of the properties of indium antimonide", Proc. Phys. Soc. (London) B 67, 775-782 (1954).

15. G.W. Charache, D.M. DePoy, J.E. Raynolds, P.F. Baldasaro, K.E. Miyano, T. Holden, F.H. Pollak, P.R. Sharps, M.L. Timmons, C.B. Geller, and W. Wolf, "Moss-Burstein and plasma reflection characteristics of heavily doped n-type $\operatorname{In}_{\mathrm{x}} \mathrm{Ga}_{1-\mathrm{x}} \mathrm{As}$ and $\mathrm{InP}_{\mathrm{y}} \mathrm{As}_{1-\mathrm{y}} "$, J. Appl. Phys. 86, 452-458 (1999).
16. J.C. Li, M. Sokolich, T. Hussain, and P.M. Asbeck, "Physical modelling of degenerately doped compound semiconductors for high-performance HBT design", Solid-State Electron. 50, 1440-1449 (2006).

17. M. Munoz, F.H. Pollak, M. Kahn, D. Ritter, L. Kronik, and G.M. Cohen, "Burstein-Moss shift of n-doped $\operatorname{In}_{0.53} \mathrm{Ga}_{0.47} \mathrm{As} /$ InP”, Phys. Rev. B 63, 233302-1-233302-3 (2001).

18. A. Tsukernik, D. Cheskis, O. Potashnik, A. Palevski, S. Bar-Ad, S. Luryi, and A. Cho, "Anomalous behaviour of the Fermi energy in heavily tin-doped InGaAs", Eur. Phys. J. B 23, 341-344 (2001). 